\title{
REDUCTION TO VERSAL DEFORMATIONS OF MATRIX PENCILS AND MATRIX PAIRS WITH APPLICATION TO CONTROL THEORY*
}

\author{
M. I. GARCÍA-PLANAS ${ }^{\dagger}$ AND A. A. MAILYBAEV $\ddagger$
}

\begin{abstract}
Matrix pencils under the strict equivalence and matrix pairs under the state feedback equivalence are considered. It is known that a matrix pencil (or a matrix pair) smoothly dependent on parameters can be reduced locally to a special typically more simple form, called the versal deformation, by a smooth change of parameters and a strict equivalence (or feedback equivalence) transformation. We suggest an explicit recurrent procedure for finding the change of parameters and equivalence transformation in the reduction of a given family of matrix pencils (or matrix pairs) to the versal deformation. As an application, this procedure is applied to the analysis of the uncontrollability set in the space of parameters for a one-input linear dynamical system. Explicit formulae for a tangent plane to the uncontrollability set at its regular point and the perturbation of the uncontrollable mode are derived. A physical example is given and studied in detail.
\end{abstract}

Key words. versal deformation, matrix pencil, matrix pair, feedback equivalence, controllability

AMS subject classifications. 15A21, 93B05, 93B52

PII. S0895479801392016

1. Introduction. The Arnold technique of constructing a local canonical form, called versal deformation, of a differentiable family of square matrices under conjugation $[1,2]$ has been generalized by several authors to matrix pencils under the strict equivalence $[4,10]$, pairs or triples of matrices under the action of the general linear group [18], pairs of matrices under the feedback similarity [6], and triples or quadruples of matrices representing linear dynamical systems under the equivalence derived from standard transformations (the change of basis in state, input, and output spaces, state feedback, and output injection) $[8,9]$. Versal deformations provide a special parametrization of matrix spaces, which can be effectively applied to perturbation analysis and investigation of complicated objects like singularities and bifurcations in multiparameter dynamical systems $[1,2,3,4,5,12,14,15]$.

The general notion of versality is the following. Let $\mathcal{M}$ be a differential manifold with the equivalence relation defined by the action of a Lie group $\mathcal{G}$. The $\mathcal{G}$-action is described by the mapping $x \longrightarrow g \circ x$, where $x, g \circ x \in \mathcal{M}$ and $g \in \mathcal{G}$. The classical example is the space of square complex matrices $\mathcal{M}=M_{m \times m}(\mathbb{C})$ with the Lie group $\mathcal{G}=\mathrm{GL}(m, \mathbb{C})$ determining the similarity transformation (the change of basis) $A \longrightarrow C^{-1} A C$, where $A \in M_{m \times m}(\mathbb{C})$ and $C \in \mathrm{GL}(m, \mathbb{C})$. Let us consider a smooth mapping $x: \mathcal{U}_{0} \longrightarrow \mathcal{M}$, where $\mathcal{U}_{0}$ is a neighborhood of the origin of the space $\mathbb{F}^{\ell} ; \mathbb{F}$ stands for the space of real or complex numbers. The mapping $x(\gamma)$ is called a deformation of $x_{0}=x(0)$ with the parameter vector $\gamma \in \mathbb{F}^{\ell}$. Introducing a change of parameters $\phi: \mathcal{U}_{0}^{\prime} \longrightarrow \mathcal{U}_{0}$, where $\mathcal{U}_{0}^{\prime}$ is a neighborhood of the origin in $\mathbb{F}^{k}$, such that $\phi(0)=0$, we obtain the deformation $x(\phi(\xi))$ of $x_{0}$ with the parameter vector

*Received by the editors July 3, 2001; accepted for publication (in revised form) by P. Van Dooren August 5, 2002; published electronically February 12, 2003. This work was supported by INTAS Young Scientists Fellowship 00-58.

http://www.siam.org/journals/simax/24-4/39201.html

${ }^{\dagger}$ Dept. de Matemàtica Aplicada I, Universitat Politècnica de Catalunya, Minería 1, Esc. C, 1-3, 08038 Barcelona, Spain (maria.isabel.garcia@upc.es).

${ }^{\ddagger}$ Institute of Mechanics, Moscow State University, Michurinsky pr. 1, 117192 Moscow, Russia (mailybaev@imec.msu.ru). 
$\xi \in \mathcal{U}_{0}^{\prime} \subset \mathbb{F}^{k}$. Applying the equivalence transformation $g(\xi)$, where $g: \mathcal{U}_{0}^{\prime} \longrightarrow \mathcal{G}$ is a smooth mapping such that $g(0)=e$ is the unit element of $\mathcal{G}$, we get the deformation

$$
z(\xi)=g(\xi) \circ x(\phi(\xi))
$$

of $z(0)=e \circ x_{0}=x_{0}$. Then $x(\gamma)$ is called a versal deformation of $x_{0}$ if any deformation $z(\xi)$ of $x_{0}$ can be represented in the form (1.1) in some neighborhood of the origin $\mathcal{U}_{0}^{\prime \prime} \subset \mathbb{F}^{k}$. This definition implies that a versal deformation generates all deformations of $x_{0}$ and, hence, possesses properties (invariant under the equivalence transformation) of all deformations of the given element $x_{0} \in \mathcal{M}$.

The theorem given by Arnold [1,2] says that the deformation $x(\gamma)$ of $x_{0}$ is versal if and only if it is transversal to the orbit of $x_{0}$ under the action of $\mathcal{G}$. This theorem reduces the problem of finding a versal deformation to solving a specific linear equation determined by $x_{0}$. This method allows finding versal deformations $x(\gamma)$ having simple form, which can be treated as local canonical forms. For the reduction of a given deformation $z(\xi)$ to this form, one needs to find the change of parameters $\gamma=\phi(\xi)$ and the equivalence transformation $g(\xi)$ smoothly depending on $\xi$, which satisfy locally equality (1.1).

In this paper versal deformations of matrix pencils under the strict equivalence and pairs of matrices under the feedback equivalence are considered. The method of finding the change of basis $\gamma=\phi(\xi)$ and the equivalence transformation $g(\xi)$, which reduce a given deformation $z(\xi)$ to the versal deformation, is developed. The mappings $\phi(\xi)$ and $g(\xi)$ are represented in the form of Taylor series, whose coefficients are found from the explicit recurrent procedure. This approach is the generalization to these particular cases of the one presented by Mailybaev $[12,13]$ for spaces of square matrices under conjugation; see also $[5,17]$ for related problems.

A pair of matrices $(F, G) \in M_{m \times m}(\mathbb{R}) \times M_{m \times n}(\mathbb{R})$ determines the linear dynamical system $\dot{\psi}=F \psi+G \nu$ with the state vector $\psi \in \mathbb{R}^{m}$ and input vector $\nu \in \mathbb{R}^{n}$. The controllability of this system (the possibility of reaching any state $\psi$ by choosing an appropriate input vector $\nu(t)$ ) is an invariant property under the feedback equivalence transformation. Using this fact, we apply the method presented in this paper to study the uncontrollability set of a multiparameter one-input linear dynamical system. As a result, explicit formulae for the tangent plane to the uncontrollability set at its regular point and the perturbation of the uncontrollable mode (the generalized eigenvalue) are derived. Note that this approach provides a simple and systematic way for the perturbation analysis of the uncontrollability set, while the classical controllability condition related to the rank of a certain matrix (called the controllability matrix) is difficult to use for multiparameter perturbation analysis.

The organization of the paper is as follows. In section 2 the case of matrix pencils under the strict equivalence is considered. The local structure of the orbit and stabilizer of a matrix pencil is described by a specific linear function (differential of the equivalence transformation mapping) and its adjoint. Using this information, a versal deformation $x(\gamma)$ is determined. Then the change of basis $\gamma=\phi(\xi)$ and the equivalence transformation $g(\xi)$ for the reduction of a given deformation $z(\xi)$ to this versal deformation are found in the form of Taylor series. Section 3 studies the case of pairs of matrices under the feedback equivalence. In section 4 the obtained results are applied to the perturbation analysis of the uncontrollability set for a one-input linear dynamical system dependent on parameters. A physical example is given and studied in detail. The conclusion discusses applicability issues of the presented method and its importance for the versal deformation theory. 
2. Matrix pencils and their deformations. Let us consider a space of matrix pencils $\mathcal{M}=\left\{A-\lambda B \mid A, B \in M_{m \times n}(\mathbb{F})\right\}$, where $M_{m \times n}(\mathbb{F})$ is a set of $m \times n$ matrices with real or complex elements, $\mathbb{F} \in\{\mathbb{R}, \mathbb{C}\}$. In this space we consider the following equivalence relation [7]: two pencils $A_{1}-\lambda B_{1}$ and $A_{2}-\lambda B_{2}$ are (strict) equivalent if and only if

$$
A_{2}-\lambda B_{2}=P^{-1}\left(A_{1}-\lambda B_{1}\right) Q
$$

for some nonsingular square matrices $P \in \mathrm{Gl}(m ; \mathbb{F}), Q \in \mathrm{Gl}(n ; \mathbb{F})$.

2.1. Equivalence as a Lie group action. Equivalence relation (2.1) may be seen as induced by the action of a Lie group $\mathcal{G}=\{(P, Q) \mid P \in \operatorname{Gl}(m ; \mathbb{F}), Q \in$ $\mathrm{Gl}(n ; \mathbb{F})\}$. Using the short notation $g=(P, Q) \in \mathcal{G}$ and $x=A-\lambda B \in \mathcal{M}$, we define multiplication in $\mathcal{G}$, action of the group $\mathcal{G}$, and equivalence condition (2.1) as follows:

$$
\begin{gathered}
g_{1} g_{2}=\left(P_{1} P_{2}, Q_{1} Q_{2}\right) \in \mathcal{G}, \\
g \circ x=P^{-1}(A-\lambda B) Q \in \mathcal{M}, \\
x_{2}=g \circ x_{1} .
\end{gathered}
$$

Multiplication in the group corresponds to successive equivalence transformations: $g_{2} \circ\left(g_{1} \circ x\right)=\left(g_{1} g_{2}\right) \circ x$. The unit element of $\mathcal{G}$ has the form $e=\left(I_{m}, I_{n}\right)$, where $I_{m}$ and $I_{n}$ are the identity matrices.

Let us fix a pencil $x_{0}=A_{0}-\lambda B_{0} \in \mathcal{M}$ and define the mapping

$$
f_{x_{0}}(g)=g \circ x_{0}
$$

The equivalence class of the pencil $x_{0}$ with respect to the action of $\mathcal{G}$ is the range of the function $f_{x_{0}}$. It is called the orbit of $x_{0}$ and denoted by

$$
\mathcal{O}\left(x_{0}\right)=\operatorname{Im} f_{x_{0}}=\left\{g \circ x_{0} \mid g \in \mathcal{G}\right\} .
$$

The stabilizer of $x_{0}$ under the $\mathcal{G}$-action is a null-space of the function $f_{x_{0}}-x_{0}$. We denote it by

$$
\mathcal{S}\left(x_{0}\right)=\operatorname{Ker}\left(f_{x_{0}}-x_{0}\right)=\left\{g \in \mathcal{G} \mid g \circ x_{0}=x_{0}\right\} .
$$

The mapping $f_{x_{0}}$ is differentiable, and $\mathcal{O}\left(x_{0}\right)$ and $\mathcal{S}\left(x_{0}\right)$ are smooth submanifolds of $\mathcal{M}$ and $\mathcal{G}$, respectively.

Let us use the notation $T_{e} \mathcal{G}$ for a tangent space to the manifold $\mathcal{G}$ at the unit element $e$. Since $\mathcal{G}$ is an open subset of $M_{m \times m}(\mathbb{F}) \times M_{n \times n}(\mathbb{F})$, we have

$$
T_{e} \mathcal{G}=\left\{(U, V) \mid U \in M_{m \times m}(\mathbb{F}), V \in M_{n \times n}(\mathbb{F})\right\}
$$

and, since $\mathcal{M}$ is a linear space,

$$
T_{x_{0}} \mathcal{M}=\mathcal{M}
$$

The Euclidean scalar products in the spaces $\mathcal{M}$ and $T_{e} \mathcal{G}$ considered in this paper are defined as follows:

$$
\begin{aligned}
& \left\langle x_{1}, x_{2}\right\rangle_{1}=\operatorname{trace}\left(A_{1} A_{2}^{*}\right)+\operatorname{trace}\left(B_{1} B_{2}^{*}\right), \quad x_{i}=A_{i}-\lambda B_{i} \in \mathcal{M}, \\
& \left\langle y_{1}, y_{2}\right\rangle_{2}=\operatorname{trace}\left(U_{1} U_{2}^{*}\right)+\operatorname{trace}\left(V_{1} V_{2}^{*}\right), \quad y_{i}=\left(U_{i}, V_{i}\right) \in T_{e} \mathcal{G},
\end{aligned}
$$



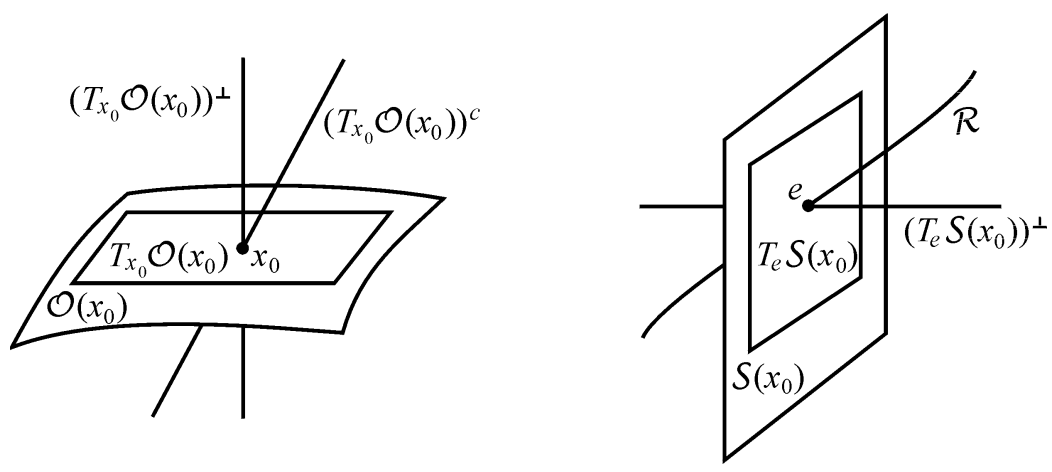

FIG. 1. Local structure of the orbit $\mathcal{O}\left(x_{0}\right)$ and stabilizer $\mathcal{S}\left(x_{0}\right)$.

where $A^{*}$ denotes the conjugate transpose of a matrix $A$.

Let $d f_{x_{0}}: T_{e} \mathcal{G} \longrightarrow \mathcal{M}$ be the differential of $f_{x_{0}}$ at the unit element $e$. Using expressions (2.2) and (2.3), we find [4]

$$
d f_{x_{0}}(y)=\left(A_{0} V-U A_{0}\right)-\lambda\left(B_{0} V-U B_{0}\right) \in \mathcal{M}, \quad y=(U, V) \in T_{e} \mathcal{G} .
$$

The adjoint linear mapping $d f_{x_{0}}^{*}: \mathcal{M} \longrightarrow T_{e} \mathcal{G}$ is defined by the relation

$$
\left\langle d f_{x_{0}}(y), z\right\rangle_{1}=\left\langle y, d f_{x_{0}}^{*}(z)\right\rangle_{2}, \quad y \in T_{e} \mathcal{G}, \quad z \in \mathcal{M} .
$$

Using expressions (2.6) and (2.7) in (2.8), it is straightforward to find

$$
d f_{x_{0}}^{*}(z)=\left(-X A_{0}^{*}-Y B_{0}^{*}, A_{0}^{*} X+B_{0}^{*} Y\right) \in T_{e} \mathcal{G}, \quad z=X-\lambda Y \in \mathcal{M} .
$$

The mappings $d f_{x_{0}}$ and $d f_{x_{0}}^{*}$ provide a simple description of the tangent spaces $T_{x_{0}} \mathcal{O}\left(x_{0}\right)$, $T_{e} \mathcal{S}\left(x_{0}\right)$ and their normal complements $\left(T_{x_{0}} \mathcal{O}\left(x_{0}\right)\right)^{\perp},\left(T_{e} \mathcal{S}\left(x_{0}\right)\right)^{\perp}$; see Figure 1.

THEOREM 2.1. The tangent spaces to the orbit and stabilizer of the matrix pencil $x_{0}$ and the corresponding normal complementary subspaces with respect to $\mathcal{M}$ and $T_{e} \mathcal{G}$ can be found in the following form:

1. $T_{x_{0}} \mathcal{O}\left(x_{0}\right)=\operatorname{Im} d f_{x_{0}} \subset \mathcal{M}$.

2. $\left(T_{x_{0}} \mathcal{O}\left(x_{0}\right)\right)^{\perp}=\operatorname{Ker} d f_{x_{0}}^{*} \subset \mathcal{M}$.

3. $T_{e} \mathcal{S}\left(x_{0}\right)=\operatorname{Ker} d f_{x_{0}} \subset T_{e} \mathcal{G}$.

4. $\left(T_{e} \mathcal{S}\left(x_{0}\right)\right)^{\perp}=\operatorname{Im} d f_{x_{0}}^{*} \subset T_{e} \mathcal{G}$.

Proof. Assertions 1 and 3 follow from (2.4), (2.5), and the definition of $d f_{x_{0}}$ as the differential of the function $f_{x_{0}}$ at $e$. Then assertions 2 and 4 follow from properties of the adjoint function $d f_{x_{0}}^{*}[7]$.

Corollary 2.2. The mappings $d f_{x_{0}}$ and $d f_{x_{0}}^{*}$ define one-to-one correspondences between the subspaces $T_{x_{0}} \mathcal{O}\left(x_{0}\right)$ and $\left(T_{e} \mathcal{S}\left(x_{0}\right)\right)^{\perp}$ :

$$
T_{x_{0}} \mathcal{O}\left(x_{0}\right) \underset{d f_{x_{0}}}{\stackrel{d f_{x_{0}}^{*}}{\rightleftarrows}}\left(T_{e} \mathcal{S}\left(x_{0}\right)\right)^{\perp}
$$

Example 2.1. Let us consider a matrix pencil

$$
x_{0}=A_{0}-\lambda B_{0}=\left(\begin{array}{cccc}
0 & 1 & 0 & 0 \\
0 & 0 & 0 & 0 \\
0 & 0 & 0 & 1
\end{array}\right)-\lambda\left(\begin{array}{cccc}
1 & 0 & 0 & 0 \\
0 & 0 & 1 & 0 \\
0 & 0 & 0 & 1
\end{array}\right) .
$$


According to Theorem 2.1, the elements $z \in\left(T_{x_{0}} \mathcal{O}\left(x_{0}\right)\right)^{\perp}$ can be found by solving the linear system $d f_{x_{0}}^{*}(z)=0$ with $d f_{x_{0}}^{*}$ given by expression (2.9). As a result, we obtain a general element of $\left(T_{x_{0}} \mathcal{O}\left(x_{0}\right)\right)^{\perp}$ in the form

$$
\left(\begin{array}{cccc}
0 & 0 & 0 & 0 \\
\gamma_{1} & 0 & \gamma_{3} & 0 \\
\gamma_{2} & \gamma_{2} & 0 & \gamma_{4}
\end{array}\right)-\lambda\left(\begin{array}{cccc}
0 & 0 & 0 & 0 \\
0 & 0 & 0 & 0 \\
-\gamma_{2} & -\gamma_{2} & 0 & -\gamma_{4}
\end{array}\right)
$$

where $\gamma_{1}, \ldots, \gamma_{4} \in \mathbb{F}$ are arbitrary; $\operatorname{dim}\left(T_{x_{0}} \mathcal{O}\left(x_{0}\right)\right)^{\perp}=4$. Using (2.11), it is straightforward to find a general element of the space $T_{x_{0}} \mathcal{O}\left(x_{0}\right)$ as follows:

$$
\left(\begin{array}{cccc}
\mu_{1} & \mu_{2} & \mu_{3} & \mu_{4} \\
0 & \mu_{5} & 0 & \mu_{6} \\
\mu_{7}+\mu_{9} & \mu_{8}-\mu_{9} & \mu_{10} & \mu_{11}
\end{array}\right)-\lambda\left(\begin{array}{cccc}
\mu_{12} & \mu_{13} & \mu_{14} & \mu_{15} \\
\mu_{16} & \mu_{17} & \mu_{18} & \mu_{19} \\
\mu_{7}-\mu_{9} & \mu_{8}+\mu_{9} & \mu_{20} & \mu_{11}
\end{array}\right)
$$

where $\mu_{1}, \ldots, \mu_{20} \in \mathbb{F}$ are arbitrary; $\operatorname{dim} T_{x_{0}} \mathcal{O}\left(x_{0}\right)=20$. Using (2.12) in Corollary 2.2 , we find a general element of the space $\left(T_{e} \mathcal{S}\left(x_{0}\right)\right)^{\perp}=d f_{x_{0}}^{*}\left(T_{x_{0}} \mathcal{O}\left(x_{0}\right)\right)$ in the form

$$
\left(\left(\begin{array}{ccc}
-\mu_{2}-\mu_{12} & -\mu_{14} & -\mu_{4}-\mu_{15} \\
-\mu_{5}-\mu_{16} & -\mu_{18} & -\mu_{6}-\mu_{19} \\
2 \mu_{9}-\mu_{8}-\mu_{7} & -\mu_{20} & -2 \mu_{11}
\end{array}\right),\left(\begin{array}{cccc}
\mu_{12} & \mu_{13} & \mu_{14} & \mu_{15} \\
\mu_{1} & \mu_{2} & \mu_{3} & \mu_{4} \\
\mu_{16} & \mu_{17} & \mu_{18} & \mu_{19} \\
2 \mu_{7} & 2 \mu_{8} & \mu_{10}+\mu_{20} & 2 \mu_{11}
\end{array}\right)\right)
$$

Finally, we obtain elements of the space $T_{e} \mathcal{S}\left(x_{0}\right)$ from the equation $d f_{x_{0}}(y)=0$ as follows:

$$
\left(\left(\begin{array}{ccc}
\nu_{1} & \nu_{2} & \nu_{3} \\
0 & \nu_{4} & 0 \\
0 & 0 & \nu_{5}
\end{array}\right),\left(\begin{array}{cccc}
\nu_{1} & 0 & \nu_{2} & \nu_{3} \\
0 & \nu_{1} & 0 & \nu_{3} \\
0 & 0 & \nu_{4} & 0 \\
0 & 0 & 0 & \nu_{5}
\end{array}\right)\right)
$$

where $\nu_{1}, \ldots, \nu_{5} \in \mathbb{F}$ are arbitrary; $\operatorname{dim} T_{e} \mathcal{S}\left(x_{0}\right)=5$.

2.2. Versal deformation. Let $\mathcal{U}_{0}$ be a neighborhood of the origin of $\mathbb{F}^{\ell}$. A deformation $x(\gamma)$ of $x_{0}$ is a smooth mapping

$$
x: \mathcal{U}_{0} \longrightarrow \mathcal{M}
$$

such that $x(0)=x_{0}$. The vector $\gamma=\left(\gamma_{1}, \ldots, \gamma_{\ell}\right) \in \mathcal{U}_{0}$ is called the parameter vector. The deformation $x(\gamma)$ is also called the family of matrix pencils. The deformation $x(\gamma)$ of $x_{0}$ is called versal if any deformation $z(\xi)$ of $x_{0}$, where $\xi=\left(\xi_{1}, \ldots, \xi_{k}\right) \in \mathcal{U}_{0}^{\prime} \subset \mathbb{F}^{k}$ is the parameter vector, can be represented in some neighborhood of the origin in the following form:

$$
z(\xi)=g(\xi) \circ x(\phi(\xi)), \quad \xi \in \mathcal{U}_{0}^{\prime \prime} \subset \mathcal{U}_{0}^{\prime},
$$

where $\phi: \mathcal{U}_{0}^{\prime \prime} \longrightarrow \mathbb{F}^{\ell}$ and $g: \mathcal{U}_{0}^{\prime \prime} \longrightarrow \mathcal{G}$ are differentiable mappings such that $\phi(0)=0$ and $g(0)=e$. Expression (2.15) means that any deformation $z(\xi)$ of $x_{0}$ can be obtained from the versal deformation $x(\gamma)$ of $x_{0}$ by an appropriate smooth change of parameters $\gamma=\phi(\xi)$ and equivalence transformation $g(\xi)$ smoothly dependent on parameters. The versal deformation with minimal possible number of parameters $\ell$ is called miniversal. 
The following result, proved by Arnold $[1,2]$ for $\operatorname{Gl}(n ; \mathbb{C})$ acting on $M_{n \times n}(\mathbb{C})$, and generalized by Tannenbaum [18] for a Lie group acting on a complex manifold, provides the relation between the versal deformation of $x_{0}$ and the local structure of the orbit and stabilizer of $x_{0}$.

THEOREM 2.3 .

1. A deformation $x(\gamma)$ of $x_{0}$ is versal if and only if it is transversal to the orbit $\mathcal{O}\left(x_{0}\right)$ at $x_{0}$.

2. The minimal number of parameters of a versal deformation is equal to the codimension of the orbit of $x_{0}$ in $\mathcal{M}, \ell=\operatorname{codim} \mathcal{O}\left(x_{0}\right)$.

3. If $x(\gamma)$ is a miniversal deformation and values of the mapping $g(\xi)$ are restricted to belong to a smooth submanifold $\mathcal{R} \subset \mathcal{G}$, which is transversal to $\mathcal{S}\left(x_{0}\right)$ at $e$ and has the minimal dimension $\operatorname{dim} \mathcal{R}=\operatorname{codim} \mathcal{S}\left(x_{0}\right)$, then the mappings $\phi(\xi)$ and $g(\xi)$ in representation (2.15) are uniquely determined by $z(\xi)$.

Note that the third assertion of Theorem 2.3 was not explicitly stated in $[1,2,18]$ but proved in the proof of the corresponding theorem.

Let us denote by $\left\{t_{1}, \ldots, t_{d}\right\}, d=\operatorname{dim} T_{x_{0}} \mathcal{O}\left(x_{0}\right)$, a basis of the tangent space $T_{x_{0}} \mathcal{O}\left(x_{0}\right)$; by $\left\{n_{1}, \ldots, n_{\ell}\right\}, \ell=\operatorname{codim} T_{x_{0}} \mathcal{O}\left(x_{0}\right)$, a basis the normal complement $\left(T_{x_{0}} \mathcal{O}\left(x_{0}\right)\right)^{\perp}$; by $\left\{c_{1}, \ldots, c_{\ell}\right\}$ a basis of an arbitrary complementary subspace $\left(T_{x_{0}} \mathcal{O}\left(x_{0}\right)\right)^{c}$ to $T_{x_{0}} \mathcal{O}\left(x_{0}\right)$; and by $\left\{r_{1}, \ldots, r_{d}\right\}$ a basis of $\left(T_{e} \mathcal{S}\left(x_{0}\right)\right)^{\perp}$. By Corollary 2.2 , if we have the basis $\left\{t_{1}, \ldots, t_{d}\right\}$, then the basis $\left\{r_{1}, \ldots, r_{d}\right\}$ can be chosen in the form $\left\{d f_{x_{0}}^{*}\left(t_{1}\right), \ldots, d f_{x_{0}}^{*}\left(t_{d}\right)\right\}$, and, vice versa, if the basis $\left\{r_{1}, \ldots, r_{d}\right\}$ is known, then we can choose the basis $\left\{t_{1}, \ldots, t_{d}\right\}$ in the form $\left\{d f_{x_{0}}\left(r_{1}\right), \ldots, d f_{x_{0}}\left(r_{d}\right)\right\}$.

Corollary 2.4. The deformation

$$
x(\gamma)=x_{0}+\sum_{i=1}^{\ell} c_{i} \gamma_{i}
$$

is a miniversal deformation. The functions $\phi(\xi)$ and $g(\xi)$ in the versal deformation reduction (2.15) are uniquely determined if the mapping $g(\xi)$ is taken in the form

$$
g(\xi)=e+\sum_{j=1}^{d} r_{j} \mu_{j}(\xi)
$$

where $\mu_{j}(\xi)$ are smooth functions in $\mathbb{F}$ such that $\mu_{j}(0)=0, j=1, \ldots, d$.

If we take $c_{i}=n_{i}, i=1, \ldots, \ell$, in (2.16), then the corresponding miniversal deformation is called orthogonal.

If the pencil $x_{0}=A_{0}-\lambda B_{0}$ is reduced to the Kronecker canonical form (this is not a restriction because of the homogeneity of the orbit), it is possible to write down explicitly the bases $\left\{c_{1}, \ldots, c_{\ell}\right\},\left\{n_{1}, \ldots, n_{\ell}\right\},\left\{t_{1}, \ldots, t_{d}\right\}$, and $\left\{r_{1}, \ldots, r_{d}\right\}$. Explicit forms of the bases $\left\{c_{1}, \ldots, c_{\ell}\right\}$ and $\left\{n_{1}, \ldots, n_{\ell}\right\}$ were given in $[4,10]$.

Example 2.2. Let us consider a matrix pencil (2.10). The matrix pencils $n_{i}, t_{j}$ and matrix pairs $r_{j}$ can be obtained from (2.11), (2.12), and (2.13), respectively, by taking $\gamma_{i}=\mu_{j}=1$ and zeros for other variables. Using the explicit form of the tangent space $T_{x_{0}} \mathcal{O}\left(x_{0}\right)$ given in (2.12), we can choose a basis $\left\{c_{1}, \ldots, c_{\ell}\right\}, \ell=4$, of a complementary subspace $\left(T_{x_{0}} \mathcal{O}\left(x_{0}\right)\right)^{c}$ such that every $c_{i}$ has exactly one nonzero element. This will give us a simplest miniversal deformation, for example,

$$
x(\gamma)=\left(\begin{array}{cccc}
0 & 1 & 0 & 0 \\
\gamma_{1} & 0 & \gamma_{3} & 0 \\
\gamma_{2} & 0 & 0 & 1+\gamma_{4}
\end{array}\right)-\lambda\left(\begin{array}{cccc}
1 & 0 & 0 & 0 \\
0 & 0 & 1 & 0 \\
0 & 0 & 0 & 1
\end{array}\right) .
$$


2.3. Reduction to miniversal deformation. Let us assume that the pencil $x_{0}$ and its miniversal deformation $x(\gamma)$ in the form (2.16) are given. To reduce an arbitrary deformation $z(\xi)$ of $x_{0}$ to the miniversal deformation, we need to find smooth mappings $\phi(\xi)$ and $g(\xi)$ satisfying relation (2.15). Recall that these mappings are unique if $g(\xi)$ is taken in the form (2.17). Since these mappings are determined in the neighborhood of the origin $\xi=0$, they can be represented in Taylor series form.

Let $h=\left(h_{1}, \ldots, h_{k}\right)$ be a vector with nonnegative integer components $h_{i} \in \mathbb{Z}_{+}$. We will use the conventional notation

$$
\begin{gathered}
|h|=h_{1}+\cdots+h_{k}, \quad h !=h_{1} ! \cdots h_{k} !, \quad C_{h}^{h^{\prime}}=\frac{h !}{h^{\prime} !\left(h-h^{\prime}\right) !}, \\
\xi^{h}=\xi_{1}^{h_{1}} \cdots \xi_{k}^{h_{k}}, \quad \phi^{(h)}=\frac{\partial^{|h|} \phi}{\partial \xi^{h_{1}} \cdots \partial \xi^{h_{k}}},
\end{gathered}
$$

where derivatives are evaluated at $\xi=0$; the derivative of zero order denotes the function value at zero, i.e., $\phi^{(0)}=\phi(0)$. Using expression (2.17), we can write the Taylor series for the mappings $\phi(\xi)$ and $g(\xi)$ as

$$
\begin{aligned}
& \phi(\xi)=\sum_{|h| \leq s} \frac{\phi^{(h)}}{h !} \xi^{h}+o\left(\|\xi\|^{s}\right), \\
& g(\xi)=e+\sum_{j=1}^{d} r_{j} \sum_{|h| \leq s} \frac{\mu_{j}^{(h)}}{h !} \xi^{h}+o\left(\|\xi\|^{s}\right),
\end{aligned}
$$

where $\phi^{(0)}=0$ and $\mu_{j}^{(0)}=0 ;\|\xi\|$ is the norm in the parameter space $\mathbb{F}^{k}$. Therefore, to find the transformation functions $\phi(\xi)$ and $g(\xi)$, we need to determine the derivatives $\phi^{(h)}=\left(\phi_{1}^{(h)}, \ldots, \phi_{\ell}^{(h)}\right)$ and $\mu_{1}^{(h)}, \ldots, \mu_{d}^{(h)}$. The following theorem provides explicit recurrent formulae for calculation of these derivatives up to an arbitrary order $|h|$.

Theorem 2.5. The derivatives $\phi_{1}^{(h)}, \ldots, \phi_{\ell}^{(h)}$ and $\mu_{1}^{(h)}, \ldots, \mu_{d}^{(h)}$ determining transformation functions (2.19), which reduce the deformation $z(\xi)$ of $x_{0}$ to the miniversal deformation (2.16), satisfy the recurrent formulae

$$
\begin{gathered}
\left(\begin{array}{c}
\phi_{1}^{(h)} \\
\vdots \\
\phi_{\ell}^{(h)}
\end{array}\right)=Z^{-1}\left(\begin{array}{c}
\left\langle s_{h}, n_{1}\right\rangle_{1} \\
\vdots \\
\left\langle s_{h}, n_{\ell}\right\rangle_{1}
\end{array}\right), \\
\left(\begin{array}{c}
\mu_{1}^{(h)} \\
\vdots \\
\mu_{d}^{(h)}
\end{array}\right)=W^{-1}\left(\begin{array}{c}
\left\langle s_{h}-\sum_{i=1}^{\ell} c_{i} \phi_{i}^{(h)}, t_{1}\right\rangle_{1} \\
\vdots \\
\left\langle s_{h}-\sum_{i=1}^{\ell} c_{i} \phi_{i}^{(h)}, t_{d}\right\rangle_{1}
\end{array}\right),
\end{gathered}
$$

where $Z$ and $W$ are nonsingular $\ell \times \ell$ and $d \times d$ matrices with the elements $z_{i j}=$ $\left\langle c_{j}, n_{i}\right\rangle_{1}$ and $w_{i j}=\left\langle d f_{x_{0}}\left(r_{j}\right), t_{i}\right\rangle_{1}=\left\langle r_{j}, d f_{x_{0}}^{*}\left(t_{i}\right)\right\rangle_{2}$, respectively. The pencil $s_{h} \in \mathcal{M}$ has the form

$$
s_{h}=z^{(h)}-\sum_{\substack{h^{\prime}+h^{\prime \prime}=h \\ h^{\prime}|>0,| h^{\prime \prime} \mid>0}} C_{h}^{h^{\prime}} \alpha\left(\sum_{i=1}^{\ell} c_{i} \phi_{i}^{\left(h^{\prime}\right)}, \sum_{j=1}^{d} r_{j} \mu_{j}^{\left(h^{\prime \prime}\right)}, z^{\left(h^{\prime}\right)}\right) .
$$


The mapping $\alpha: \mathcal{M} \times T_{e} \mathcal{G} \times \mathcal{M} \longrightarrow \mathcal{M}$ is defined by the expression

$$
\alpha(x, y, z)=(A V-U X)-\lambda(B V-U Y),
$$

where $x=A-\lambda B, y=(U, V)$, and $z=X-\lambda Y$.

Proof. Using the notation $x=A-\lambda B, g=(P, Q)$, and $z=X-\lambda Y$, we can write expression (2.15) in the form

$$
X(\xi)-\lambda Y(\xi)=P^{-1}(\xi)(A(\phi(\xi))-\lambda B(\phi(\xi))) Q(\xi) .
$$

Multiplying (2.24) by $P(\xi)$ from left and collecting all terms at the left-hand side, we obtain

$$
P(\xi)(X(\xi)-\lambda Y(\xi))-(A(\phi(\xi))-\lambda B(\phi(\xi))) Q(\xi)=0 .
$$

Taking the derivative of order $h$ of $(2.25)$ and using the Leibniz formula for differentiation of a function product, we get

$$
\begin{gathered}
\sum_{h^{\prime}+h^{\prime \prime}=h} C_{h}^{h^{\prime}}\left[P^{\left(h^{\prime \prime}\right)}\left(X^{\left(h^{\prime}\right)}-\lambda Y^{\left(h^{\prime}\right)}\right)\right. \\
\left.-\left((A(\phi(\xi)))^{\left(h^{\prime}\right)}-\lambda(B(\phi(\xi)))^{\left(h^{\prime}\right)}\right) Q^{\left(h^{\prime \prime}\right)}\right]=0 .
\end{gathered}
$$

Using expressions (2.16), (2.17), (2.22), (2.23) in (2.26) and taking into account that $P^{(0)}=I_{m}, Q^{(0)}=I_{n}, A^{(0)}=X^{(0)}=A_{0}, B^{(0)}=Y^{(0)}=B_{0}$, after permutation of terms we find

$$
d f_{x_{0}}\left(\sum_{j=1}^{d} r_{j} \mu_{j}^{(h)}\right)=s_{h}-\sum_{i=1}^{\ell} c_{i} \phi_{i}^{(h)},
$$

where the linear mapping $d f_{x_{0}}$ is defined in (2.7).

Equality (2.27) represents a system of linear equations with respect to $\ell+d=2 m n$ unknowns $\phi_{1}^{(h)}, \ldots, \phi_{\ell}^{(h)}$ and $\mu_{1}^{(h)}, \ldots, \mu_{d}^{(h)}$. The solution of (2.27) exists if and only if its right-hand side belongs to $\operatorname{Im} d f_{x_{0}}=T_{x_{0}} \mathcal{O}\left(x_{0}\right)$. Hence, the right-hand side has to be orthogonal to every pencil from the basis $\left\{n_{1}, \ldots, n_{\ell}\right\}$ of $\left(T_{x_{0}} \mathcal{O}\left(x_{0}\right)\right)^{\perp}$. This condition, written in the matrix form, yields

$$
\left(\begin{array}{c}
\left\langle s_{h}-\sum_{i=1}^{\ell} c_{i} \phi_{i}^{(h)}, n_{1}\right\rangle_{1} \\
\vdots \\
\left\langle s_{h}-\sum_{i=1}^{\ell} c_{i} \phi_{i}^{(h)}, n_{\ell}\right\rangle_{1}
\end{array}\right)=\left(\begin{array}{c}
\left\langle s_{h}, n_{1}\right\rangle_{1} \\
\vdots \\
\left\langle s_{h}, n_{\ell}\right\rangle_{1}
\end{array}\right)-Z\left(\begin{array}{c}
\phi_{1}^{(h)} \\
\vdots \\
\phi_{\ell}^{(h)}
\end{array}\right)=0 .
$$

The solution of this system gives expression (2.20) of the theorem.

To determine values of the derivatives $\mu_{1}^{(h)}, \ldots, \mu_{d}^{(h)}$, we take the scalar product of $(2.27)$ and $t_{i}$. For the left-hand side this yields

$$
\left\langle d f_{x_{0}}\left(\sum_{j=1}^{d} r_{j} \mu_{j}^{(h)}\right), t_{i}\right\rangle_{1}=\sum_{j=1}^{d}\left\langle d f_{x_{0}}\left(r_{j}\right), t_{i}\right\rangle_{1} \mu_{j}^{(h)}=\sum_{j=1}^{d} w_{i j} \mu_{j}^{(h)} .
$$

Recall that $\left\langle d f_{x_{0}}\left(r_{j}\right), t_{i}\right\rangle_{1}=\left\langle r_{j}, d f_{x_{0}}^{*}\left(t_{i}\right)\right\rangle_{2}$ by definition (2.8). Taking $i=1, \ldots, d$, we obtain the following system of linear equations:

$$
W\left(\begin{array}{c}
\mu_{1}^{(h)} \\
\vdots \\
\mu_{d}^{(h)}
\end{array}\right)=\left(\begin{array}{c}
\left\langle s_{h}-\sum_{i=1}^{\ell} c_{i} \phi_{i}^{(h)}, t_{1}\right\rangle_{1} \\
\vdots \\
\left\langle s_{h}-\sum_{i=1}^{\ell} c_{i} \phi_{i}^{(h)}, t_{d}\right\rangle_{1}
\end{array}\right)
$$


The solution of (2.30) gives expression (2.21) of the theorem.

Note that for evaluation of derivatives $\phi_{i}^{(h)}, \mu_{j}^{(h)}$, expressions of Theorem 2.5 require only derivatives $\phi_{i}^{\left(h^{\prime}\right)}, \mu_{j}^{\left(h^{\prime}\right)}$ of lower orders $\left|h^{\prime}\right|<|h|$ and derivatives $z^{\left(h^{\prime}\right)}$ of orders $\left|h^{\prime}\right|<|h|$ and $h^{\prime}=h$. This makes it possible to use Theorem 2.5 for successive calculation of the derivatives $\phi_{i}^{(h)}, \mu_{j}^{(h)}$ in order to find the transformation functions $\phi(\xi)$ and $g(\xi)$ in the form of Taylor series (2.19) up to small terms of arbitrary order. Recall that at the initial step of the recurrent procedure we take $\phi_{i}^{(0)}=0$ and $\mu_{j}^{(0)}=0$.

The matrices $Z^{-1}$ and $W^{-1}$ have to be computed only once in the beginning of the recurrent procedure. The size $d$ of the matrix $W$ is typically close to $2 m n$ and can be big. Nevertheless, this matrix is usually very sparse. Moreover, we can avoid difficulties with the inversion by making the matrices $Z$ and $W$ diagonal. For this purpose, we need to choose the bases $\left\{c_{1}, \ldots, c_{\ell}\right\},\left\{n_{1}, \ldots, n_{\ell}\right\},\left\{t_{1}, \ldots, t_{d}\right\}$, and $\left\{r_{1}, \ldots, r_{d}\right\}$ such that $\left\langle c_{j}, n_{i}\right\rangle_{1}=0$ and $\left\langle d f_{x_{0}}\left(r_{j}\right), t_{i}\right\rangle_{1}=0$ for $i \neq j$.

Note that the orthogonal miniversal deformation, represented by the orthonormal basis $\left\{n_{1}, \ldots, n_{\ell}\right\}$ of $\left(T_{x_{0}} \mathcal{O}\left(x_{0}\right)\right)^{\perp}$, keeps the metric information in the normal direction to the orbit $\mathcal{O}\left(x_{0}\right)$. This deformation is useful for the numerical problem of computation of a Kronecker canonical form [4]. In many applications, a metric based on properties of the underlying system is defined in the parameter space rather than in the whole space of matrix pencils. Computation on the mapping $\gamma=\phi(\xi)$ connecting the parameter spaces allows us to keep the metric information of the original parameter space and transfer this metric into the parameter space of the miniversal deformation. Theorem 2.5 can be used with an arbitrary versal deformation satisfying the requirements of each particular problem.

As noted by Arnold [1, 2], a miniversal deformation can be chosen in a simple form, which makes it convenient for applications. To avoid numerical instability in transformation to the miniversal deformation, the angle between the image of the miniversal deformation $x(\gamma)$ and the tangent space to the orbit $T_{x_{0}} \mathcal{O}\left(x_{0}\right)$ should not be small, i.e., the transversality condition of Theorem 2.3 should not be affected by numerical uncertainties and round-off.

Example 2.3. Let us consider the following two-parameter deformation $z(\xi), \xi=$ $\left(\xi_{1}, \xi_{2}\right)$, of matrix pencil $(2.10)$ :

$$
z(\xi)=\left(\begin{array}{cccc}
\sin \xi_{1} & 1 & 0 & \xi_{2}^{2} \\
0 & \sin \xi_{2} & \xi_{2} & \xi_{1} \\
\xi_{1} \xi_{2} & \sin \xi_{1} & 0 & \cos \xi_{2}
\end{array}\right)-\lambda\left(\begin{array}{cccc}
1 & 0 & 0 & \xi_{1} \xi_{2} \\
0 & \sin \xi_{2} & 1+\xi_{2} & \xi_{1} \\
\xi_{2}^{2} & 0 & \xi_{2} & \cos \xi_{1}
\end{array}\right)
$$

Using the pencils $c_{1}, \ldots, c_{4}, n_{1}, \ldots, n_{4}, t_{1}, \ldots, t_{20}$ and pairs $r_{1}, \ldots, r_{20}$, constructed in Examples 2.1, 2.2, and applying Theorem 2.5, we find

$$
\begin{aligned}
\phi_{1}(\xi) & =-\xi_{1} \xi_{2}+\xi_{2}^{2}+\xi_{1}^{3}-2 \xi_{1}^{2} \xi_{2}+\frac{3}{2} \xi_{1} \xi_{2}^{2}-\frac{5}{2} \xi_{2}^{3}+o\left(\|\xi\|^{3}\right), \\
\phi_{2}(\xi) & =\xi_{1}-\xi_{1}^{2}+\xi_{1} \xi_{2}-\xi_{2}^{2}+\frac{7}{12} \xi_{1}^{3}+\frac{1}{2} \xi_{1}^{2} \xi_{2}-\frac{1}{2} \xi_{1} \xi_{2}^{2}+o\left(\|\xi\|^{3}\right), \\
\phi_{3}(\xi) & =\xi_{2}-\xi_{2}^{2}+\xi_{1} \xi_{2}^{2}+\xi_{2}^{3}+o\left(\|\xi\|^{3}\right), \\
\phi_{4}(\xi) & =\frac{1}{2} \xi_{1}^{2}-\frac{1}{2} \xi_{2}^{2}+\frac{1}{3} \xi_{1}^{2} \xi_{2}-\frac{4}{6} \xi_{1} \xi_{2}^{2}+o\left(\|\xi\|^{3}\right), \\
\mu_{1}(\xi) & =\xi_{1}-\frac{1}{6} \xi_{1}^{3}+o\left(\|\xi\|^{3}\right) \\
\mu_{2}(\xi) & =-\frac{2}{9} \xi_{1}^{2} \xi_{2}-\frac{2}{9} \xi_{1} \xi_{2}^{2}+o\left(\|\xi\|^{3}\right)
\end{aligned}
$$




$$
\begin{aligned}
& \mu_{3}(\xi)=o\left(\|\xi\|^{3}\right) \\
& \mu_{4}(\xi)=-\frac{1}{3} \xi_{1} \xi_{2}+\frac{2}{3} \xi_{2}^{2}+o\left(\|\xi\|^{3}\right) \\
& \mu_{5}(\xi)=2 \xi_{2}-2 \xi_{1}^{2}-3 \xi_{2}^{2}+\xi_{1}^{2} \xi_{2}+2 \xi_{1} \xi_{2}^{2}+\frac{19}{6} \xi_{2}^{3}+o\left(\|\xi\|^{3}\right), \\
& \mu_{6}(\xi)=\xi_{1}-\frac{1}{2} \xi_{1} \xi_{2}-\frac{1}{2} \xi_{1}^{3}+\frac{1}{2} \xi_{1}^{2} \xi_{2}+\frac{9}{4} \xi_{1} \xi_{2}^{2}-2 \xi_{2}^{3}+o\left(\|\xi\|^{3}\right), \\
& \mu_{7}(\xi)=-\frac{1}{2} \xi_{1}+\frac{1}{2} \xi_{2}^{2}-\frac{1}{24} \xi_{1}^{3}-\frac{1}{4} \xi_{1}^{2} \xi_{2}+o\left(\|\xi\|^{3}\right), \\
& \mu_{8}(\xi)=-\frac{1}{2} \xi_{2}^{2}+o\left(\|\xi\|^{3}\right) \\
& \mu_{9}(\xi)=-\frac{3}{4} \xi_{1}-\frac{1}{16} \xi_{1}^{3}-\frac{3}{8} \xi_{1}^{2} \xi_{2}+o\left(\|\xi\|^{3}\right), \\
& \mu_{10}(\xi)=-\xi_{2}-\xi_{2}^{2}-\frac{1}{4} \xi_{1}^{2} \xi_{2}-\frac{1}{2} \xi_{1} \xi_{2}^{2}+o\left(\|\xi\|^{3}\right) \\
& \mu_{11}(\xi)=-\frac{1}{8} \xi_{1}^{2}-\frac{1}{4} \xi_{1} \xi_{2}-\frac{1}{4} \xi_{1}^{2} \xi_{2}+o\left(\|\xi\|^{3}\right), \\
& \mu_{12}(\xi)=\frac{1}{9} \xi_{1}^{2} \xi_{2}+\frac{1}{9} \xi_{1} \xi_{2}^{2}+o\left(\|\xi\|^{3}\right), \\
& \mu_{13}(\xi)=o\left(\|\xi\|^{3}\right) \text {, } \\
& \mu_{14}(\xi)=-\frac{1}{6} \xi_{1} \xi_{2}^{2}-\frac{1}{6} \xi_{2}^{3}+o\left(\|\xi\|^{3}\right) \\
& \mu_{15}(\xi)=\frac{2}{3} \xi_{1} \xi_{2}-\frac{1}{3} \xi_{2}^{2}+o\left(\|\xi\|^{3}\right), \\
& \mu_{16}(\xi)=-\xi_{2}+\xi_{1}^{2}+\frac{3}{2} \xi_{2}^{2}-\frac{1}{2} \xi_{1}^{2} \xi_{2}-\frac{3}{2} \xi_{1} \xi_{2}^{2}-\frac{19}{12} \xi_{2}^{3}+o\left(\|\xi\|^{3}\right), \\
& \mu_{17}(\xi)=\xi_{2}-\frac{1}{2} \xi_{2}^{2}+\frac{1}{2} \xi_{1} \xi_{2}^{2}+\frac{1}{12} \xi_{2}^{3}+o\left(\|\xi\|^{3}\right), \\
& \mu_{18}(\xi)=\frac{1}{2} \xi_{2}-\frac{1}{2} \xi_{1} \xi_{2}-\frac{1}{4} \xi_{2}^{2}+\frac{1}{2} \xi_{1} \xi_{2}^{2}+\frac{1}{8} \xi_{2}^{3}+o\left(\|\xi\|^{3}\right), \\
& \mu_{19}(\xi)=\frac{1}{2} \xi_{1}^{3}-\frac{3}{2} \xi_{1} \xi_{2}^{2}+\xi_{2}^{3}+o\left(\|\xi\|^{3}\right), \\
& \mu_{20}(\xi)=\xi_{2}+\frac{1}{4} \xi_{1}^{2} \xi_{2}+\frac{1}{2} \xi_{1} \xi_{2}^{2}+o\left(\|\xi\|^{3}\right) \text {. }
\end{aligned}
$$

These expressions determine the change of parameters $\gamma=\phi(\xi)$ and equivalence transformation $g(\xi)$ in the reduction of $z(\xi)$ to the miniversal deformation (2.18).

3. Pairs of matrices under the feedback equivalence. In this section we consider the space of pairs of matrices

$$
\widetilde{\mathcal{M}}=\left\{(F, G) \mid F \in M_{m \times m}(\mathbb{F}), \quad G \in M_{m \times n}(\mathbb{F})\right\} .
$$

Each pair $x=(F, G) \in \widetilde{M}$ represents the time-invariant linear dynamical system $\dot{\psi}=F \psi+G \nu, \psi \in \mathbb{F}^{m}$, with the input vector $\nu \in \mathbb{F}^{n}$. The change of basis in the state and input spaces and feedback operation in this system induce an equivalence relation in the space $\widetilde{\mathcal{M}}$ as follows: two pairs of matrices $x_{1}=\left(F_{1}, G_{1}\right)$ and $x_{2}=\left(F_{2}, G_{2}\right)$ are called feedback equivalent if and only if there exist matrices $P \in \operatorname{Gl}(m ; \mathbb{F}), R \in$ $\mathrm{Gl}(n ; \mathbb{F})$, and $S \in M_{n \times m}(\mathbb{F})$ such that [16]

$$
F_{2}=P^{-1}\left(F_{1} P+G_{1} S\right), \quad G_{2}=P^{-1} G_{1} R .
$$


The feedback equivalence transformation may be seen as the action of the Lie group

$$
\widetilde{\mathcal{G}}=\left\{g=(P, R, S) \mid P \in \mathrm{Gl}(m ; \mathbb{F}), R \in \mathrm{Gl}(n ; \mathbb{F}), S \in M_{n \times m}(\mathbb{F})\right\}
$$

with the multiplication of elements $g_{1}, g_{2} \in \widetilde{\mathcal{G}}$ determined by the expression

$$
g_{1} g_{2}=\left(P_{1} P_{2}, R_{1} R_{2}, S_{1} P_{2}+R_{1} S_{2}\right), \quad g_{i}=\left(P_{i}, R_{i}, S_{i}\right) .
$$

The unit element of the group $\widetilde{\mathcal{G}}$ is $e=\left(I_{m}, I_{n}, 0\right)$. We will use the short notation $x_{2}=g \circ x_{1}$ for the equivalence relation (3.2). Note that $g_{1} g_{2} \circ x=g_{2} \circ\left(g_{1} \circ x\right)$.

Given a pair of matrices $x=(F, G) \in \widetilde{\mathcal{M}}$ and a triple $g=(P, R, S) \in \widetilde{\mathcal{G}}$, we can associate a matrix pencil $x^{\prime} \in \mathcal{M}$ of dimension $m \times(m+n)$ and a pair $g^{\prime}$ from the corresponding Lie group $\mathcal{G}$ in the following manner:

$$
x^{\prime}=\left(\begin{array}{ll}
F & G
\end{array}\right)-\lambda\left(\begin{array}{ll}
I_{m} & 0
\end{array}\right), \quad g^{\prime}=\left(P,\left(\begin{array}{cc}
P & 0 \\
S & R
\end{array}\right)\right) .
$$

It is easy to see that $x_{2}=g \circ x_{1}$ (the pairs $x_{1}$ and $x_{2}$ are feedback equivalent) if and only if $x_{2}^{\prime}=g^{\prime} \circ x_{1}^{\prime}$ (the associated matrix pencils $x_{1}^{\prime}$ and $x_{2}^{\prime}$ are strict equivalent) [11]. Hence, $\widetilde{\mathcal{M}}$ and $\widetilde{\mathcal{G}}$ can be seen as the subspace of $\mathcal{M}$ and subgroup of $\mathcal{G}$, respectively. Note that the subspace $\widetilde{\mathcal{M}} \subset \mathcal{M}$ is not invariant under the action of the Lie group $\mathcal{G}$ defined over the space of matrix pencils.

3.1. Orbit and stabilizer. Let us fix some pair of matrices $x_{0}=\left(F_{0}, G_{0}\right)$ and define the mapping $\widetilde{f}_{x_{0}}(g)=g \circ x_{0}, g \in \widetilde{\mathcal{G}}$. Then the orbit $\widetilde{\mathcal{O}}\left(x_{0}\right)$ and stabilizer $\widetilde{\mathcal{S}}\left(x_{0}\right)$ of the pair $x_{0}$ are defined as follows:

$$
\begin{gathered}
\widetilde{\mathcal{O}}\left(x_{0}\right)=\operatorname{Im} \widetilde{f}_{x_{0}}=\left\{g \circ x_{0} \mid g \in \widetilde{\mathcal{G}}\right\} \\
\widetilde{\mathcal{S}}\left(x_{0}\right)=\operatorname{Ker}\left(\widetilde{f}_{x_{0}}-x_{0}\right)=\left\{g \in \widetilde{\mathcal{G}} \mid g \circ x_{0}=x_{0}\right\} .
\end{gathered}
$$

The sets $\widetilde{\mathcal{O}}\left(x_{0}\right)$ and $\widetilde{\mathcal{S}}\left(x_{0}\right)$ are differentiable submanifolds of $\widetilde{\mathcal{M}}$ and $\widetilde{\mathcal{G}}$, respectively. Note that under relations $(3.5)$ we have $\widetilde{\mathcal{O}}\left(x_{0}\right) \subset \mathcal{O}\left(x_{0}\right)$ and $\widetilde{\mathcal{S}}\left(x_{0}\right) \subset \mathcal{S}\left(x_{0}\right)$.

Since $\widetilde{\mathcal{G}}$ is an open subset of $M_{m \times m}(\mathbb{F}) \times M_{n \times n}(\mathbb{F}) \times M_{n \times m}(\mathbb{F})$, the tangent space $T_{e} \widetilde{\mathcal{G}}$ to the manifold $\widetilde{\mathcal{G}}$ at the unit element $e$ is

$$
T_{e} \widetilde{\mathcal{G}}=\left\{(U, V, W) \mid U \in M_{m \times m}(\mathbb{F}), V \in M_{n \times n}(\mathbb{F}), W \in M_{n \times m}(\mathbb{F})\right\} .
$$

Since $\widetilde{\mathcal{M}}$ is a linear space, $T_{x_{0}} \widetilde{\mathcal{M}}=\widetilde{\mathcal{M}}$. We consider Euclidean scalar products in $\widetilde{\mathcal{M}}$ and $T_{e} \widetilde{\mathcal{G}}$ having the form

$$
\begin{aligned}
& \left\langle x_{1}, x_{2}\right\rangle_{1}=\operatorname{trace}\left(F_{1} F_{2}^{*}\right)+\operatorname{trace}\left(G_{1} G_{2}^{*}\right), \\
& \left\langle y_{1}, y_{2}\right\rangle_{2}=\operatorname{trace}\left(U_{1} U_{2}^{*}\right)+\operatorname{trace}\left(V_{1} V_{2}^{*}\right)+\operatorname{trace}\left(W_{1} W_{2}^{*}\right),
\end{aligned}
$$

where $x_{i}=\left(F_{i}, G_{i}\right) \in \widetilde{\mathcal{M}}, y_{i}=\left(U_{i}, V_{i}, W_{i}\right) \in T_{e} \widetilde{\mathcal{G}}, i=1,2$.

Let $d \widetilde{f}_{x_{0}}: T_{e} \widetilde{\mathcal{G}} \longrightarrow \widetilde{\mathcal{M}}$ be the differential of $\widetilde{f}_{x_{0}}$ at the unit element $e$. Using (3.2), it can be shown [6] that

$$
d \widetilde{f}_{x_{0}}(y)=\left(F_{0} U-U F_{0}+G_{0} W, G_{0} V-U G_{0}\right) \in \widetilde{\mathcal{M}},
$$


where $y=(U, V, W) \in T_{e} \widetilde{\mathcal{G}}$. The adjoint linear mapping $d \tilde{f}_{x_{0}}^{*}: \widetilde{\mathcal{M}} \longrightarrow T_{e} \widetilde{\mathcal{G}}$ is determined by the relation

$$
d \tilde{f}_{x_{0}}^{*}(z)=\left(F_{0}^{*} X-X F_{0}^{*}-Y G_{0}^{*}, G_{0}^{*} Y, G_{0}^{*} X\right) \in T_{e} \widetilde{\mathcal{G}},
$$

where $z=(X, Y) \in \widetilde{\mathcal{M}}$.

Analogously to Theorem 2.1, the mappings $d \widetilde{f}_{x_{0}}$ and $d \widetilde{f}_{x_{0}}^{*}$ provide the following description for the tangent spaces $T_{x_{0}} \widetilde{\mathcal{O}}\left(x_{0}\right), T_{e} \widetilde{\mathcal{S}}\left(x_{0}\right)$ and their normal complements.

THEOREM 3.1. The tangent spaces to the orbit and stabilizer of the pair of matrices $x_{0}$ and corresponding normal complementary subspaces can be found in the following form:

1. $T_{x_{0}} \widetilde{\mathcal{O}}\left(x_{0}\right)=\operatorname{Im} d \widetilde{f}_{x_{0}} \subset \widetilde{\mathcal{M}}$.

2. $\left(T_{x_{0}} \widetilde{\mathcal{O}}\left(x_{0}\right)\right)^{\perp}=\operatorname{Ker} d \widetilde{f}_{x_{0}}^{*} \subset \widetilde{\mathcal{M}}$.

3. $T_{e} \widetilde{\mathcal{S}}\left(x_{0}\right)=\operatorname{Ker} d \widetilde{f}_{x_{0}} \subset T_{e} \widetilde{\mathcal{G}}$.

4. $\left(T_{e} \widetilde{\mathcal{S}}\left(x_{0}\right)\right)^{\perp}=\operatorname{Im} d \widetilde{f}_{x_{0}}^{*} \subset T_{e} \widetilde{\mathcal{G}}$.

Example 3.1. Let $x_{0}=\left(F_{0}, G_{0}\right)$ be a pair of matrices with

$$
F_{0}=\left(\begin{array}{ccc}
0 & 0 & 0 \\
0 & 0 & 0 \\
0 & 0 & 1
\end{array}\right), \quad G_{0}=\left(\begin{array}{l}
1 \\
0 \\
0
\end{array}\right) .
$$

Then elements $z=(X, Y)$ of the space $\left(T_{x_{0}} \widetilde{\mathcal{O}}\left(x_{0}\right)\right)^{\perp}$ can be found from the equation $d \widetilde{f}_{x_{0}}^{*}(z)=0$ in the form

$$
\left(\left(\begin{array}{ccc}
0 & 0 & 0 \\
\gamma_{1} & \gamma_{2} & 0 \\
\gamma_{3} & 0 & \gamma_{4}
\end{array}\right),\left(\begin{array}{c}
0 \\
0 \\
\gamma_{3}
\end{array}\right)\right)
$$

where $\gamma_{1}, \ldots, \gamma_{4} \in \mathbb{F}$ are arbitrary, and $\operatorname{dim}\left(T_{x_{0}} \widetilde{\mathcal{O}}\left(x_{0}\right)\right)^{\perp}=4$. The elements of $T_{x_{0}} \widetilde{\mathcal{O}}\left(x_{0}\right)$ have the form

$$
\left(\left(\begin{array}{ccc}
\mu_{1} & \mu_{2} & \mu_{3} \\
0 & 0 & \mu_{4} \\
\mu_{5} & \mu_{6} & 0
\end{array}\right),\left(\begin{array}{c}
\mu_{7} \\
\mu_{8} \\
-\mu_{5}
\end{array}\right)\right),
$$

where $\mu_{1}, \ldots, \mu_{8} \in \mathbb{F}$ are arbitrary and $\operatorname{dim} T_{x_{0}} \widetilde{\mathcal{O}}\left(x_{0}\right)=8$. Then, by Theorem 3.1, $\operatorname{dim}\left(T_{e} \widetilde{\mathcal{S}}\left(x_{0}\right)\right)^{\perp}=8$ and elements $y=(U, V, W)$ of $\left(T_{e} \widetilde{\mathcal{S}}\left(x_{0}\right)\right)^{\perp}=d \widetilde{f}_{x_{0}}^{*}\left(T_{x_{0}} \widetilde{\mathcal{O}}\left(x_{0}\right)\right)$ take the form

$$
\left(\left(\begin{array}{ccc}
-\mu_{7} & 0 & -\mu_{3} \\
-\mu_{8} & 0 & -\mu_{4} \\
2 \mu_{5} & \mu_{6} & 0
\end{array}\right),\left(\mu_{7}\right),\left(\mu_{1}, \mu_{2}, \mu_{3}\right)\right) .
$$

Finally, the space $T_{e} \widetilde{\mathcal{S}}\left(x_{0}\right)=\operatorname{Ker} d \widetilde{f}_{x_{0}}$ is formed by the triples

$$
\left(\left(\begin{array}{ccc}
\nu_{1} & \nu_{2} & \nu_{3} \\
0 & \nu_{4} & 0 \\
0 & 0 & \nu_{5}
\end{array}\right),\left(\nu_{1}\right),\left(0,0, \nu_{3}\right)\right)
$$

where $\nu_{1}, \ldots, \nu_{5} \in \mathbb{F}$ are arbitrary and $\operatorname{dim} T_{e} \widetilde{\mathcal{S}}\left(x_{0}\right)=5$. 
Note that under relation (3.5), the matrix pencil corresponding to pair (3.12) is equivalent to matrix pencil (2.10) considered in Example 2.1. Dimensions of the tangent space to the stabilizer and normal complement of the tangent space to the orbit are the same for the cases of matrix pairs and matrix pencils. But dimensions of the tangent space to the orbit and the normal complement of the tangent space to the stabilizer are smaller in the case of matrix pairs.

3.2. Versal deformation. Let us consider a deformation $x(\gamma)$ of $x_{0} \in \widetilde{\mathcal{M}}$ in the form

$$
x(\gamma)=x_{0}+\sum_{i=1}^{\ell} c_{i} \gamma_{i}
$$

where $\left\{c_{1}, \ldots, c_{\ell}\right\}$ is a basis of an arbitrary complementary subspace $\left(T_{x_{0}} \widetilde{\mathcal{O}}\left(x_{0}\right)\right)^{c}$ to $T_{x_{0}} \widetilde{\mathcal{O}}\left(x_{0}\right) ; \ell=\operatorname{codim} T_{x_{0}} \widetilde{\mathcal{O}}\left(x_{0}\right)$.

Analogously to Corollary 2.4, we have the following.

Corollary 3.2. The deformation (3.17) is a miniversal deformation; i.e., any deformation $z(\xi), \xi \in \mathbb{F}^{k}$, of $x_{0}$ can be represented in the neighborhood of the origin $\mathcal{U}_{0} \subset \mathbb{F}^{k}$ in the form

$$
z(\xi)=g(\xi) \circ x(\phi(\xi))
$$

where $\phi: \mathcal{U}_{0} \longrightarrow \mathbb{F}^{\ell}$ and $g: \mathcal{U}_{0} \longrightarrow \widetilde{\mathcal{G}}$ are smooth mappings such that $\phi(0)=0$ and $g(0)=e$. The functions $\phi(\xi)$ and $g(\xi)$ are uniquely determined by the deformation $z(\xi)$ if $g(\xi)$ is taken in the form

$$
g(\xi)=e+\sum_{j=1}^{d} r_{j} \mu_{j}(\xi)
$$

where $\mu_{j}(\xi)$ are smooth functions in $\mathbb{F}$ such that $\mu_{j}(0)=0, j=1, \ldots, d$, and $\left\{r_{1}, \ldots, r_{d}\right\}$ is a basis of $\left(T_{e} \widetilde{\mathcal{S}}\left(x_{0}\right)\right)^{\perp}$.

Recall that if $\left\{t_{1}, \ldots, t_{d}\right\}$ is a basis of $T_{x_{0}} \widetilde{\mathcal{O}}\left(x_{0}\right)$, then $\left\{d \widetilde{f}_{x_{0}}^{*}\left(t_{1}\right), \ldots, d \widetilde{f}_{x_{0}}^{*}\left(t_{d}\right)\right\}$ is a basis of $\left(T_{e} \widetilde{\mathcal{S}}\left(x_{0}\right)\right)^{\perp}$, and, vice versa, if $\left\{r_{1}, \ldots, r_{d}\right\}$ is a basis of $\left(T_{e} \widetilde{\mathcal{S}}\left(x_{0}\right)\right)^{\perp}$, then $\left\{d \widetilde{f}_{x_{0}}\left(r_{1}\right), \ldots, d \widetilde{f}_{x_{0}}\left(r_{d}\right)\right\}$ is a basis of $T_{x_{0}} \widetilde{\mathcal{O}}\left(x_{0}\right)$.

For pairs of matrices, reduced to the Brunovsky canonical form, explicit expressions for the bases $\left\{c_{1}, \ldots, c_{\ell}\right\}$ and $\left\{n_{1}, \ldots, n_{\ell}\right\}$ may be found in [6].

Example 3.2. Let $x_{0}=\left(F_{0}, G_{0}\right)$ be the pair of matrices considered in Example 3.1. Using explicit form of the tangent space $T_{x_{0}} \widetilde{\mathcal{O}}\left(x_{0}\right)$ given in (3.14), we can choose a basis $\left\{c_{1}, \ldots, c_{4}\right\}$ of the complementary space $\left(T_{x_{0}} \widetilde{\mathcal{O}}\left(x_{0}\right)\right)^{c}$ such that every $c_{i}$ has exactly one nonzero element. For example, we can choose the miniversal deformation in the form

$$
x(\gamma)=\left(\left(\begin{array}{ccc}
0 & 0 & 0 \\
\gamma_{1} & \gamma_{2} & 0 \\
\gamma_{3} & 0 & 1+\gamma_{4}
\end{array}\right),\left(\begin{array}{l}
1 \\
0 \\
0
\end{array}\right)\right), \quad \gamma=\left(\gamma_{1}, \ldots, \gamma_{4}\right) .
$$

3.3. Reduction to miniversal deformation. Let $x_{0}$ and $x(\gamma)$ be a pair of matrices and its miniversal deformation. In order to reduce a given deformation $z(\xi)$ 
of $x_{0}$ to the miniversal deformation, we need to find the smooth mappings $\phi(\xi)$ and $g(\xi)$ satisfying (3.18). These mappings can be found in Taylor series form:

$$
\begin{aligned}
& \phi(\xi)=\sum_{|h| \leq s} \frac{\phi^{(h)}}{h !} \xi^{h}+o\left(\|\xi\|^{s}\right), \\
& g(\xi)=e+\sum_{j=1}^{d} r_{j} \sum_{|h| \leq s} \frac{\mu_{j}^{(h)}}{h !} \xi^{h}+o\left(\|\xi\|^{s}\right),
\end{aligned}
$$

where $\phi^{(0)}=0$ and $\mu_{j}^{(0)}=0$.

Analogously to Theorem 2.5, we can find explicit recurrent formulae for calculation of the derivatives $\phi^{(h)}$ and $\mu_{j}^{(h)}$ up to an arbitrary order.

THEOREM 3.3. The derivatives $\phi_{1}^{(h)}, \ldots, \phi_{\ell}^{(h)}$ and $\mu_{1}^{(h)}, \ldots, \mu_{d}^{(h)}$ determining transformation functions (3.21), which reduce the deformation $z(\xi)$ of $x_{0}$ to the miniversal deformation (3.17), satisfy the recurrent formulae

$$
\begin{gathered}
\left(\begin{array}{c}
\phi_{1}^{(h)} \\
\vdots \\
\phi_{\ell}^{(h)}
\end{array}\right)=Z^{-1}\left(\begin{array}{c}
\left\langle s_{h}, n_{1}\right\rangle_{1} \\
\vdots \\
\left\langle s_{h}, n_{\ell}\right\rangle_{1}
\end{array}\right), \\
\left(\begin{array}{c}
\mu_{1}^{(h)} \\
\vdots \\
\mu_{d}^{(h)}
\end{array}\right)=W^{-1}\left(\begin{array}{c}
\left\langle s_{h}-\sum_{i=1}^{\ell} c_{i} \phi_{i}^{(h)}, t_{1}\right\rangle_{1} \\
\vdots \\
\left\langle s_{h}-\sum_{i=1}^{\ell} c_{i} \phi_{i}^{(h)}, t_{d}\right\rangle_{1}
\end{array}\right),
\end{gathered}
$$

where $Z$ and $W$ are nonsingular $\ell \times \ell$ and $d \times d$ matrices with the elements $z_{i j}=$ $\left\langle c_{j}, n_{i}\right\rangle_{1}, w_{i j}=\left\langle d \widetilde{f}_{x_{0}}\left(r_{j}\right), t_{i}\right\rangle_{1}$, respectively. The pair of matrices $s_{h} \in \widetilde{\mathcal{M}}$ has the form

$$
s_{h}=z^{(h)}-\sum_{\substack{h^{\prime}+h^{\prime \prime}=h \\\left|h^{\prime}\right|>0,\left|h^{\prime \prime}\right|>0}} C_{h}^{h^{\prime}} \widetilde{\alpha}\left(\sum_{i=1}^{\ell} c_{i} \phi_{i}^{\left(h^{\prime}\right)}, \sum_{j=1}^{d} r_{j} \mu_{j}^{\left(h^{\prime \prime}\right)}, z^{\left(h^{\prime}\right)}\right) .
$$

The mapping $\widetilde{\alpha}: \widetilde{\mathcal{M}} \times T_{e} \widetilde{\mathcal{G}} \times \widetilde{\mathcal{M}} \longrightarrow \widetilde{\mathcal{M}}$ is defined as follows:

$$
\widetilde{\alpha}(x, y, z)=(F U-U X+G W, G V-U Y),
$$

where $x=(F, G), y=(U, V, W)$, and $z=(X, Y)$.

Analogously to the case of matrix pencils, in order to simplify the calculations we can choose the bases $\left\{c_{1}, \ldots, c_{\ell}\right\},\left\{n_{1}, \ldots, n_{\ell}\right\},\left\{t_{1}, \ldots, t_{d}\right\}$, and $\left\{r_{1}, \ldots, r_{d}\right\}$ in such a way that $\left\langle c_{j}, n_{i}\right\rangle_{1}=0$ and $\left\langle d \widetilde{f}_{x_{0}}\left(r_{j}\right), t_{i}\right\rangle_{1}=0$ for $i \neq j$, which implies that $Z$ and $W$ are diagonal matrices.

Example 3.3. Let us consider the following two-parameter deformation $z(\xi), \xi=$ $\left(\xi_{1}, \xi_{2}\right)$, of the pair of matrices $x_{0}=\left(F_{0}, G_{0}\right)$ considered in Example 3.1:

$$
z(\xi)=\left(\left(\begin{array}{ccc}
\xi_{1} & \xi_{1} \xi_{2} & \xi_{2}^{3} / 6 \\
\xi_{2} & \xi_{1} & \xi_{1}+\xi_{2} \\
\xi_{1}^{2} \xi_{2} & \xi_{1} \xi_{2} & 1
\end{array}\right),\left(\begin{array}{c}
1+\xi_{1} \xi_{2} \\
\xi_{1}^{2} \\
\xi_{2}^{3}
\end{array}\right)\right) .
$$


Using the bases $\left\{c_{1}, \ldots, c_{4}\right\},\left\{n_{1}, \ldots, n_{4}\right\},\left\{t_{1}, \ldots, t_{8}\right\}$, and $\left\{r_{1}, \ldots, r_{8}\right\}$ constructed in Examples 3.1, 3.2 and applying Theorem 3.3, we find

$$
\begin{aligned}
& \phi_{1}(\xi)=\xi_{2}+\xi_{1} \xi_{2}^{2} / 2+o\left(\|\xi\|^{3}\right), \quad \phi_{2}(\xi)=\xi_{1}-\xi_{1}^{2} \xi_{2}-\xi_{1} \xi_{2}^{2}+o\left(\|\xi\|^{3}\right), \\
& \phi_{3}(\xi)=\xi_{1}^{2} \xi_{2}+\xi_{1} \xi_{2}^{2}+\xi_{2}^{3}+o\left(\|\xi\|^{3}\right), \quad \phi_{4}(\xi)=\xi_{1}^{2} \xi_{2}+\xi_{1} \xi_{2}^{2}+o\left(\|\xi\|^{3}\right) \\
& \mu_{1}(\xi)=\xi_{1}-\xi_{1}^{2} \xi_{2} / 2+o\left(\|\xi\|^{3}\right), \quad \mu_{2}(\xi)=\xi_{1} \xi_{2}+o\left(\|\xi\|^{3}\right) \\
& \mu_{3}(\xi)=\xi_{2}^{3} / 12+o\left(\|\xi\|^{3}\right), \\
& \mu_{4}(\xi)=\xi_{1}+\xi_{2}+\xi_{1}^{2}+\xi_{1} \xi_{2}+\xi_{1}^{3}+\xi_{1}^{2} \xi_{2}+o\left(\|\xi\|^{3}\right), \\
& \mu_{5}(\xi)=-\xi_{2}^{3} / 2+o\left(\|\xi\|^{3}\right), \quad \mu_{6}(\xi)=\xi_{1} \xi_{2}+\xi_{1}^{2} \xi_{2}+o\left(\|\xi\|^{3}\right) \\
& \mu_{7}(\xi)=\xi_{1} \xi_{2} / 2+o\left(\|\xi\|^{3}\right), \quad \mu_{8}(\xi)=\xi_{1}^{2}+o\left(\|\xi\|^{3}\right) .
\end{aligned}
$$

Expressions (3.27) determine the change of parameters $\gamma=\phi(\xi)$ and the equivalence transformation $g(\xi)$ given by (3.19) in the reduction of $z(\xi)$ to the miniversal deformation (3.20).

4. Local analysis of the uncontrollability set for one-input systems. Let us consider a pair of real matrices $z=(F, G) \in \widehat{\mathcal{M}}$ with $n=1$ and arbitrary $m$. This pair corresponds to the system of differential equations

$$
\dot{\psi}(t)=F \psi(t)+G \nu(t)
$$

with $m$-dimensional state vector $\psi \in \mathbb{R}^{m}$ and one input variable $\nu \in \mathbb{R}$. System (4.1) is called controllable if it is possible to construct a control signal $\nu(t)$ that will transfer an initial state to any final state in finite time [16]. The pair $z=(F, G)$ corresponding to such a system is called controllable. The well-known criterion for controllability says that the pair $z$ is controllable if and only if the controllability matrix $C=\left[G, F G, \ldots, F^{m-1} G\right]$ has full rank $[16]$

$$
\operatorname{rank}\left[G, F G, \ldots, F^{m-1} G\right]=m .
$$

For one-input systems, i.e., when the matrix $G$ has dimension $m \times 1$, this criterion takes the form

$$
\operatorname{det}\left[G, F G, \ldots, F^{m-1} G\right] \neq 0 \text {. }
$$

Let us consider a family of matrix pairs $z(\xi)=(F(\xi), G(\xi))$ with the parameter vector $\xi \in \mathbb{R}^{k}$. The set of values of the parameter vector $\xi$ such that the pair $z(\xi)$ is uncontrollable is called the uncontrollability set and will be denoted by $\mathcal{N}=\{\xi \in$ $\left.\mathbb{R}^{k} \mid \operatorname{rank} C(\xi)<m\right\}$. Let us assume that the pair $z(\xi)$ is uncontrollable at some point $\xi_{0} \in \mathcal{N}$. We are going to analyze the structure of the uncontrollability set in the neighborhood of this point. Due to the complicated entry of elements of the matrices $F$ and $G$ into the controllability matrix, it is difficult to use the controllability condition (4.3) for analytical analysis of the set $\mathcal{N}$. Using reduction of the family $z(\xi)$ to the miniversal deformation, this analysis can be carried out in a more simple and systematic way, as shown below.

The matrix pair $z_{0}=z\left(\xi_{0}\right)$ can be reduced to the Brunovsky canonical form $\widehat{z}_{0}=g_{0} \circ z_{0}$ by the state feedback transformation $g_{0} \in \widetilde{\mathcal{G}}[11,16]$. Let us consider the 
case when the Brunovsky form $\widehat{z}_{0}$ is as follows:

$$
\widehat{z}_{0}=\left(\left(\begin{array}{ccccc}
0 & 1 & & & \\
& 0 & \ddots & & \\
& & \ddots & 1 & \\
& & & 0 & \\
& & & & \sigma_{0}
\end{array}\right),\left(\begin{array}{c}
0 \\
\vdots \\
0 \\
1 \\
0
\end{array}\right)\right)
$$

where $\sigma_{0} \in \mathbb{R}$ is an arbitrary number called the uncontrollable mode or the generalized eigenvalue. In the generic (typical) case, the parameter vectors $\xi$, corresponding to the matrix pairs $z(\xi)$ having Brunovsky form (4.4), represent typical elements of the uncontrollability set $\mathcal{N}$ and form a codimension 1 smooth submanifold of $\mathbb{R}^{k}$. Uncontrollable matrix pairs having different Brunovsky structures form submanifolds of higher codimensions [6]. The following proposition gives explicit formulae for the tangent plane to the uncontrollability set $\mathcal{N}$ at $\xi_{0}$ and the first approximation of the uncontrollable mode.

Proposition 4.1. Let $z_{0}=z\left(\xi_{0}\right), \xi_{0} \in \mathcal{N}$, be a matrix pair having Brunovsky canonical form (4.4) with the triple $g_{0}=\left(P_{0}, R_{0}, S_{0}\right) \in \widetilde{\mathcal{G}}$ providing the feedback equivalence transformation $\widehat{z}_{0}=g_{0} \circ z_{0}$. Let us define real vectors $\eta=\left(\eta_{1}, \ldots, \eta_{k}\right)$ and $\eta_{\sigma}=\left(\eta_{\sigma 1}, \ldots, \eta_{\sigma k}\right)$ with the components

$$
\begin{aligned}
& \eta_{i}=P_{0}^{-1}(m,:)\left[\frac{\partial F}{\partial \xi_{i}} \sum_{j=1}^{m-1} \sigma_{0}^{j-1} P_{0}(:, j)+\frac{\partial G}{\partial \xi_{i}}\left(\sum_{j=1}^{m-1} \sigma_{0}^{j-1} S_{0}(:, j)+\sigma_{0}^{m-1} R_{0}\right)\right], \\
& \eta_{\sigma i}=P_{0}^{-1}(m,:)\left(\frac{\partial F}{\partial \xi_{i}} P_{0}(:, m)+\frac{\partial G}{\partial \xi_{i}} S_{0}(:, m)\right), \quad i=1, \ldots, k,
\end{aligned}
$$

where $P_{0}^{-1}(m,:), P_{0}(:, j)$, and $S_{0}(:, j)$ denote the $m$ th row of $P_{0}^{-1}$, the $j$ th column of $P_{0}$, and the jth column of $S_{0}$, respectively. Then, if $\eta \neq 0$, the uncontrollability set $\mathcal{N}$ is a smooth hypersurface in the vicinity of $\xi_{0}$; the vector $\eta$ is the normal vector to this hypersurface at $\xi_{0}$; the tangent plane to $\mathcal{N}$ at $\xi_{0}$ is given by the equation

$$
\left(\eta, \xi-\xi_{0}\right)=0
$$

where $(\eta, \xi)=\sum_{i=1}^{k} \eta_{i} \xi_{i}$ is a scalar product in $\mathbb{R}^{k}$; and the first order approximation of the uncontrollable mode on the hypersurface $\mathcal{N}$ is given by the relation

$$
\sigma(\xi)=\sigma_{0}+\left(\eta_{\sigma}, \xi-\xi_{0}\right)+o\left(\left\|\xi-\xi_{0}\right\|\right)
$$

Proof. Without loss of generality, we can take $\xi_{0}=0$. Let us consider the family $\widehat{z}(\xi)=g_{0} \circ z(\xi)$, which is a deformation of the matrix $\widehat{z}_{0}=g_{0} \circ z_{0}$ given by (4.4). The deformation $\widehat{z}(\xi)$ can be reduced to the orthogonal miniversal deformation of $\widehat{z}_{0}$ having the form $[6]$

$$
x(\gamma)=\left(\left(\begin{array}{ccccc}
0 & 1 & & & \\
& 0 & \ddots & & \\
& & \ddots & 1 & \\
& & & 0 & \\
\gamma_{1} & \sigma_{0} \gamma_{1} & \cdots & \sigma_{0}^{m-2} \gamma_{1} & \sigma_{0}+\gamma_{2}
\end{array}\right),\left(\begin{array}{c}
0 \\
\vdots \\
0 \\
1 \\
\sigma_{0}^{m-1} \gamma_{1}
\end{array}\right)\right),
$$


where $\gamma=\left(\gamma_{1}, \gamma_{2}\right)$. Since the controllability property is invariant under the feedback group action [16], the controllability of the pair $z(\xi)$ is equivalent to the controllability of the pair $x(\phi(\xi))$, where $\gamma=\phi(\xi)$ represents the change of parameters in the reduction of $\widehat{z}(\xi)$ to the miniversal deformation $x(\gamma)$. Applying the criterion of controllability (4.2) to matrix pair (4.8), we conclude that for small $\gamma$ the pair $x(\gamma)$ is controllable if and only if $\gamma_{1} \neq 0$. Hence, the uncontrollability set in the vicinity of $\xi_{0}$ is determined by the equation $\gamma_{1}=\phi_{1}(\xi)=0$. If $\gamma_{1}=0$, then we find the uncontrollable mode $\sigma=\sigma_{0}+\gamma_{2}=\sigma_{0}+\phi_{2}(\xi)$.

Using formula (3.22) of Theorem 3.3 and taking into account that the matrix $Z$ is diagonal, we find

$$
\begin{gathered}
\frac{\partial \phi_{1}}{\partial \xi_{i}}=z_{11}^{-1}\left\langle\frac{\partial \widehat{z}}{\partial \xi_{i}}, n_{1}\right\rangle_{1}=z_{11}^{-1}\left(\sum_{j=1}^{m-1} \sigma_{0}^{j-1} \frac{\partial \widehat{F}_{m j}}{\partial \xi_{i}}+\sigma_{0}^{m-1} \frac{\partial \widehat{G}_{m 1}}{\partial \xi_{i}}\right), \\
z_{11}=\left\langle n_{1}, n_{1}\right\rangle_{1}=1+\sigma_{0}^{2}+\cdots+\sigma_{0}^{2 m-2},
\end{gathered}
$$

where derivatives are taken at $\xi_{0}$, the pair $n_{1}$ was found from the orthogonal miniversal deformation (4.8) as a coefficient corresponding to $\gamma_{1}$, and $\widehat{F}_{m j}, \widehat{G}_{m 1}$ denote the $(m, j)$ th and $(m, 1)$ th elements of the matrices $(\widehat{F}, \widehat{G})=\widehat{z}$. Using expression $\widehat{z}(\xi)=$ $g_{0} \circ z(\xi)$, we obtain

$$
\widehat{F}(\xi)=P_{0}^{-1}\left(F(\xi) P_{0}+G(\xi) S_{0}\right), \quad \widehat{G}(\xi)=P_{0}^{-1} G(\xi) R_{0} .
$$

Substitution of (4.10) into (4.9) yields

$$
\begin{aligned}
\frac{\partial \phi_{1}}{\partial \xi_{i}} & =z_{11}^{-1} P_{0}^{-1}(m,:)\left[\frac{\partial F}{\partial \xi_{i}} \sum_{j=1}^{m-1} \sigma_{0}^{j-1} P_{0}(:, j)\right. \\
& \left.+\frac{\partial G}{\partial \xi_{i}}\left(\sum_{j=1}^{m-1} \sigma_{0}^{j-1} S_{0}(:, j)+\sigma_{0}^{m-1} R_{0}\right)\right] .
\end{aligned}
$$

Hence, using the notation of (4.5), we find the gradient vector of the function $\phi_{1}(\xi)$ at $\xi_{0}$ in the form

$$
\nabla \phi_{1}=\left(\frac{\partial \phi_{1}}{\partial \xi_{1}}, \ldots, \frac{\partial \phi_{1}}{\partial \xi_{k}}\right)=z_{11}^{-1} \eta
$$

If $\eta \neq 0$, then $\nabla \phi_{1} \neq 0$ and, by the implicit function theorem applied to the equation $\phi_{1}(\xi)=0$, we conclude that the uncontrollability set is a smooth hypersurface in the vicinity of $\xi_{0}$ with the tangent plane (4.6). The vector $\eta$ is normal to this surface at $\xi_{0}$.

Analogously, we find

$$
\begin{gathered}
\frac{\partial \phi_{2}}{\partial \xi_{i}}=z_{22}^{-1}\left\langle\frac{\partial \widehat{z}}{\partial \xi_{i}}, n_{2}\right\rangle_{1}=\frac{\partial \widehat{F}_{m m}}{\partial \xi_{i}} \\
=P_{0}^{-1}(m,:)\left(\frac{\partial F}{\partial \xi_{i}} P_{0}(:, m)+\frac{\partial G}{\partial \xi_{i}} S_{0}(:, m)\right) .
\end{gathered}
$$

Hence, using the notation of (4.5), we find the gradient $\nabla \phi_{2}=\eta_{\sigma}$ at $\xi_{0}$, which gives approximation (4.7) for the uncontrollable mode $\sigma(\xi)=\sigma_{0}+\phi_{2}(\xi)$. 




FIG. 2. Elastic system controlled by a force $F$.

Note that Proposition 4.1 provides quantitative local information on the uncontrollability set using only information on the matrix pair $z_{0}=z\left(\xi_{0}\right)$ and derivatives of the system matrices $F(\xi)$ and $G(\xi)$ evaluated at the point $\xi_{0}$. Using this information we can choose an optimal change of parameters in order to obtain a good-controllable system. Formula for the tangent plane is useful for numerical computation of the uncontrollability set.

A multi-input system is characterized by a vector of real input variables $\nu(t)$ in (4.1). In this case uncontrollable pairs have different Brunovsky forms, and corresponding miniversal deformations are more complicated. The suggested approach can be extended to analysis of the uncontrollability set for a multi-input dynamical system depending on parameters. For this purpose, we need to find the uncontrollability set for that particular versal deformation, and then transfer the result to the original parameter space by means of the mapping $\gamma=\phi(\xi)$ found by Theorem 2.5.

Example 4.1. Let us consider the mechanical system shown in Figure 2. The system consists of a light platform of length $L$ carrying a point mass $m$ in the middle; both ends of the platform are supported on the ground by means of springs with elastic coefficients $k_{1}, k_{2}$ and damping coefficients $c_{1}, c_{2}$. The system is controlled by a force $F$ applied to the platform at the distance $\xi_{1} L$ from the left end. We assume that the equilibrium of this system for $F=0$ corresponds to the horizontal position of the platform. Equations of motion of the system have the form

$$
\begin{aligned}
& m\left(\ddot{x}_{1}+\ddot{x}_{2}\right) / 4+c_{1} \dot{x}_{1}+k_{1} x_{1}=\left(1-\xi_{1}\right) F \\
& m\left(\ddot{x}_{1}+\ddot{x}_{2}\right) / 4+c_{2} \dot{x}_{2}+k_{2} x_{2}=\xi_{1} F
\end{aligned}
$$

where $x_{1}$ and $x_{2}$ are vertical displacements of the left and right ends of the platform, respectively. Taking $m=1, c_{1}=c_{2}=1, k_{1}=\xi_{2}, k_{2}=\xi_{3}, F=\nu$ and introducing new state variables $\psi_{1}=x_{1}+x_{2}, \psi_{2}=\dot{\psi}_{1}, \psi_{3}=x_{2}$, after simple manipulations we obtain system (4.1), depending on the vector of parameters $\xi \in \mathbb{R}^{3}$ with one control variable $\nu$, the state vector $\psi \in \mathbb{R}^{3}$, and the matrices

$$
F(\xi)=\left(\begin{array}{ccc}
0 & 1 & 0 \\
-2 \xi_{2} & -2 & 2\left(\xi_{2}-\xi_{3}\right) \\
\xi_{2} / 2 & 1 / 2 & -\left(\xi_{2}+\xi_{3}\right) / 2
\end{array}\right), \quad G(\xi)=\left(\begin{array}{c}
0 \\
2 \\
\xi_{1}-1 / 2
\end{array}\right)
$$

Let us consider a point $\xi_{0}=(1 / 4,3 / 2,5 / 6)$ in the parameter space. At this point 


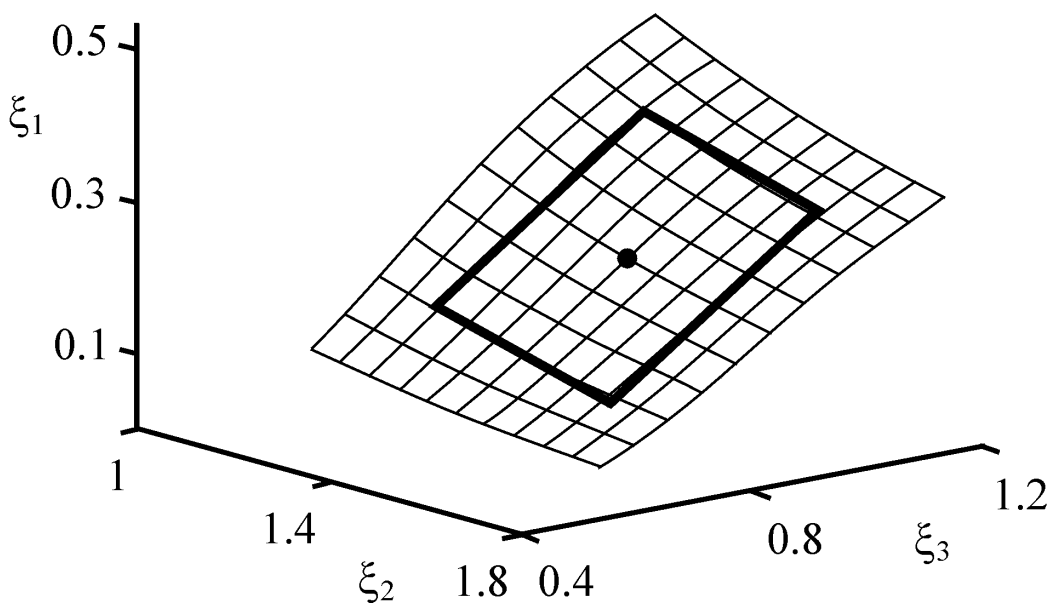

FIG. 3. Uncontrollability set and its tangent plane.

the pair of matrices (4.15) takes the form

$$
F_{0}=\left(\begin{array}{ccc}
0 & 1 & 0 \\
-3 & -2 & 4 / 3 \\
3 / 4 & 1 / 2 & -7 / 6
\end{array}\right), \quad G_{0}=\left(\begin{array}{c}
0 \\
2 \\
-1 / 4
\end{array}\right)
$$

It is straightforward to check that the pair $\left(F_{0}, G_{0}\right)$ is uncontrollable and can be transformed to the Brunovsky form (4.4) with $\sigma_{0}=-1$ by the triple $\left(P_{0}, R_{0}, S_{0}\right) \in \widetilde{\mathcal{G}}$ of the following form:

$$
P_{0}=\left(\begin{array}{ccc}
1 & 0 & 0 \\
0 & 1 & 0 \\
3 / 8 & -1 / 8 & 1
\end{array}\right), \quad R_{0}=1 / 2, \quad S_{0}=(5 / 4,13 / 12,-2 / 3)
$$

Using (4.15) and (4.17) in (4.5), we find

$$
\eta=(2 / 3,1 / 8,-3 / 8), \quad \eta_{\sigma}=-(2 / 3,1 / 4,3 / 4) .
$$

Hence, by Proposition 4.1, the uncontrollability set is a smooth hypersurface in the vicinity of $\xi_{0}$. The tangent plane to this surface at $\xi_{0}$ is given by the equation

$$
\left(\eta, \xi-\xi_{0}\right)=\frac{2 \xi_{1}}{3}+\frac{\xi_{2}}{8}-\frac{3 \xi_{3}}{8}-\frac{1}{24}=0,
$$

and the perturbation of the uncontrollable mode on this surface has the form

$$
\sigma(\xi)=-1-\frac{2\left(\xi_{1}-1 / 4\right)}{3}-\frac{\xi_{2}-3 / 2}{4}-\frac{3\left(\xi_{3}-5 / 6\right)}{4}+o\left(\left\|\xi-\xi_{0}\right\|\right) .
$$

The plane (4.19) is plotted in Figure 3 (bold rectangular). For comparison, the uncontrollability set found numerically using (4.3) (determinant of the controllability matrix changes the sign when we cross the uncontrollability set) is shown in Figure 3. Numerical computations confirm the analytical results. 
5. Conclusion. The general idea of any normal form theory is to transform an object under consideration to a form whose properties are easy to analyze. In this process both the normal form and transformation to it are important. For example, the Jordan normal form of a square matrix determines its spectrum, while knowledge of the transformation to the Jordan form (change of basis) allows us to find explicitly a general solution to the corresponding dynamical system.

In this paper we have solved the second part of the normal form problem (finding the transformation) in the reduction of families of matrix pencils and matrix pairs to the local normal form (miniversal deformation). Information on the transformation (the change of parameters and equivalence transformation) allows the development of the multi-parameter perturbation theory for multi-input linear dynamical systems. In a similar problem for square matrices, advantages of this approach for the perturbation analysis of the spectrum and stability of linear dynamical systems depending on parameters have been illustrated in $[3,12,14,15]$. In section 4 of this paper it has been shown that the suggested method is useful for the controllability analysis of single-input dynamical systems dependent on parameters.

Acknowledgment. The second author thanks M. I. García-Planas for the hospitality during his staying at the Department of Applied Mathematics I, UPC, Barcelona.

\section{REFERENCES}

[1] V. I. Arnold, On matrices depending on parameters, Russian Math. Surveys, 26 (1971), pp. 29-43.

[2] V. I. ARnold, Geometrical Methods in the Theory of Ordinary Differential Equations, Springer-Verlag, New York, 1988.

[3] J. V. Burke AND M. L. Overton, Stable perturbations of nonsymmetric matrices, Linear Algebra Appl., 171 (1992), pp. 249-273.

[4] A. Edelman, E. Elmroth, and B. K̊̊gström, A geometric approach to perturbation theory of matrices and matrix pencils. Part I: Versal deformations, SIAM J. Matrix Anal. Appl., 18 (1997), pp. 653-692.

[5] A. Edelman and Y. MA, Staircase failures explained by orthogonal versal forms, SIAM J. Matrix Anal. Appl., 21 (2000), pp. 1004-1025.

[6] J. Ferrer, M. I. Garcia-Planas, and F. Puerta, Brunowsky local form of a holomorphic family of pairs of matrices, Linear Algebra Appl., 253 (1997), pp. 175-198.

[7] F. R. Gantmacher, The Theory of Matrices, Vols. 1, 2, Chelsea, New York, 1959.

[8] M. I. Garcia-Planas and M. D. Magret, Deformation and stability of triples of matrices, Linear Algebra Appl., 254 (1997), pp. 159-192.

[9] M. I. Garcia-Planas and M. D. Magret, Miniversal deformations of linear systems under the full group action, System Control Lett., 35 (1998), pp. 279-286.

[10] M. I. Garcia-Planas and V. V. Sergeichuk, Simplest miniversal deformations of matrices, matrix pencils, and contragredient matrix pencils, Linear Algebra Appl., 302-303 (1999), pp. $45-61$.

[11] I. Gohberg, P. Lancaster, and L. Rodman, Invariant Subspaces of Matrices with Applications, Chelsea, New York, 1977.

[12] A. A. Mallybaev, Transformation of families of matrices to normal forms and its application to stability theory, SIAM J. Matrix Anal. Appl., 21 (1999), pp. 396-417.

[13] A. A. Mailybaev, Transformation to versal deformations of matrices, Linear Algebra Appl., 337 (2001), pp. 87-108.

[14] A. A. Mallybaev, On stability domains of nonconservative systems under small parametric excitation, Acta Mechanica, 154 (2002), pp. 11-30.

[15] A. A. Mailybaev and A. P. Seyranian, On singularities of a boundary of the stability domain, SIAM J. Matrix Anal. Appl., 21 (1999), pp. 106-128.

[16] E. D. Sontag, Mathematical Control Theory: Deterministic Finite Dimensional Systems, Texts Appl. Math. 6, Springer-Verlag, New York, 1990.

[17] L. Stolovitch, On the computation of a versal family of matrices, Numer. Algorithms, 4 (1993), pp. 25-46.

[18] A. Tannenbaum, Invariance and System Theory: Algebraic and Geometric Aspects, Lecture Notes in Math. 845, Springer-Verlag, New York, 1981. 